\title{
LA TRASHUMANCIA DEL OVINO SEGUREÑO: UNA OPORTUNIDAD PARA LA INNOVACIÓN DOCENTE EN VETERINARIA
}

Segureño ovine transhumance: an opportunity for veterinary innovation teaching

\author{
Contreras, A. . ${ }^{\text {; }}$ Sánchez López, A.1; Corrales, J.C. . ; García-Galán, A. . ; García Romero, E. ' \\ De la Fe, C. ${ }^{1}$; Aldeguer, M.P.'; Gómez-Martín, A. ${ }^{3}$;
}

${ }^{1}$ Departamento de Sanidad Animal, Facultad de Veterinaria de Murcia, Campus de Espinardo, 30100 Espinardo, Murcia, España. ${ }^{2}$ Departamento de Ciencias Sociosanitarias, Facultad de Veterinaria de Murcia, Campus de Espinardo, 30100 Espinardo, Murcia, España. ${ }^{3}$ Grupo de Investigación Agentes Microbiológicos Asociados a la Reproducción Animal (ProVaginBio). Departamento de Sanidad Animal y Salud Pública, Facultad de Veterinaria de la Universidad CEU Cardenal Herrera de Valencia, CEU Universities. España.

* Autor para correspondencia: Antonio Contreras. Email: acontrer@um.es Tel: 868 884730, Fax: 868884147.

Historial del artículo:

Recibido: 3 de febrero, 2020

Aceptado: 9 de octubre, 2020

\section{RESUMEN}

La importancia de la producción de cordero en el Sureste, la existencia de un núcleo de trashumantes de ovino en la Sierra de Segura, y la consideración de la trashumancia como manifestación representativa del patrimonio cultural inmaterial nos ha llevado a explorar el potencial docente de la trashumancia con ovino segureño para estudiantes de veterinaria. Entre 2016 y 2019, cuatro profesores y dieciocho estudiantes de veterinaria de las Universidades de Murcia y Cardenal Herrera (UCH-CEU) de Valencia, hemos realizado diecinueve jornadas de trashumancia. Entre las acciones de innovación docente generadas destaca la exposición fotográfica "La Vereda de los Pontoneros" y el documental "Trashumancia, la Última Frontera". Proponemos la integración de una semana de trashumancia en el periodo de las dos semanas de prácticas tuteladas con la Asociación Nacional de Criadores de Ovino Segureño (ANCOS), discutimos su integración en el grado de Veterinaria de Murcia y evidenciamos el interés docente de esta actividad inmersiva, que supera los propios contenidos del grado de veterinaria.

Palabras clave: trashumancia, oveja segureña, inmersión docente, veterinaria. 


\section{SUMMARY}

The importance of lamb production in the Southeast, the existence of ovine trashumants in Sierra de Segura and the consideration of transhumance as a representative manifestation of the intangible cultural heritage has leaded us to explore the teaching potential of transhumance with segureño sheep for veterinary students. Between 2016 and 2018, four professors and eighteen veterinary students from the University of Murcia and the University CEU Cardenal Herrera of Valencia participated during nineteen days in transhumance. Within the generated teaching innovation activities highlights the photographic exposition "La Vereda de los Pontoneros" and the documentary "Trashumancia, la última frontera". We propose the integration of a week of transhumance within the practical period with the Asociación Nacional de Criadores de Ovino Segureño (ANCOS), we discuss its inclusion in the Veterinary Degree program of the University of Murcia and we evidence the teaching interest of this immersive activity, which widely approach others fields than the veterinary.

Key words: ovine, segureño, transhumance, inmersive, teaching, veterinary.

\section{INTRODUCCIÓN}

En la Región Murcia se mantiene una ganadería ovina extensiva, de aptitud cárnica, que aprovecha recursos naturales mediante el pastoreo de razas autóctonas, como la segureña y en menor medida la montesina. La importancia de esta ganadería extensiva se incrementa por su carácter sostenible, el valor patrimonial y genético de estas razas autóctonas y el carácter trashumante del ovino explotado en la Sierra de Segura, lo que supone una responsabilidad formativa, además de una magnífica oportunidad para la Facultad de Veterinaria de Murcia. El objetivo general de este artículo es explorar el potencial docente de la realización de prácticas con ovino segureño extensivo y trashumante en nuestro entorno. Entre los objetivos específicos del trabajo se encuentra el intento de reforzar el conocimiento, por parte de los futuros profesionales veterinarios, de los valores de la ganadería extensiva y trashumante que podrían contribuir a la mejora de un sector ganadero en crisis, el de la ganadería extensiva ovina.

\section{LA GANADERÍA OVINA AUTÓCTONA DEL SURESTE}

La Región de Murcia tiene una importante vocación de producción de cordero segureño, con una marcada producción extensiva en la comarca del Noroeste. Con respecto a la raza pura de ovino segureño, los últimos datos oficiales (MAPA 2018) concluyen que el $18 \%$ de los animales y el $9 \%$ de las explotaciones se ubican en Murcia, estando el $79 \%$ y el $87 \%$, respectivamente, en Andalucía. La raza segureña destaca por su adaptación a las duras condiciones de las Cordilleras Béticas Orientales y por la alta calidad y sabor de su carne. Además del segureño producido en la Región de Murcia, la Sierra de Segura alberga una notable cabaña cuyo núcleo principal se ubica en la comarca de Santiago-Pontones (Jaén), explotada mayoritariamente en régimen ecológico y sometida a un régimen trashumante. La conservación de las razas autóctonas es una responsabilidad y una obligación, no sólo por su valor cultural y genético, sino por su capacidad de adaptabilidad a las condiciones ambientales del territorio.

Los sistemas de producción ovina anteriormente definidos están considerados por la Red Nacional de Granjas Típicas del sector ovino de carne español como una de las unidades objeto del proyecto, la denominada "Unidad 3: Valle (Andalucía oriental y Murcia)" (RENGRATI, 2018). Su manejo reproductivo predominante es de 3 partos en 2 años, con un destete a los 45 días (16 kg) y cebo hasta los 22-25 kg (75-90 días), mientras que los valores medios en la Región de Murcia son de un 
destete a 50 días $(15 \mathrm{~kg})$ y cebo hasta los 22,5 $\mathrm{kg}$ (rango 18-26 kg) (Vacas, 2018). Los sistemas productivos considerados como los más representativos del ovino segureño en extensivo (Puntas, 2019) son el cerealista (practicado en gran parte de la comarca del Noroeste de Murcia), el de sierra (realizado por las explotaciones trashumantes de la Sierra de Segura) y el hortícola, mayoritariamente con pastoreo conducido, que es el que se practica en gran parte de la región de Murcia para aprovechar recursos forrajeros de la agricultura.

Gracias a iniciativas generadas por la Asociación Nacional de Criadores de Ovino Segureño (ANCOS), se dispone de una Identificación Geográfica Protegida (IGP) del Cordero Segureño, marca de calidad diferenciada que define sus cualidades y origen. Así, la IGP del Segureño garantiza al consumidor la procedencia del cordero de rebaños de esta raza y procedentes de un origen común, definido por una altitud mínima de $500 \mathrm{~m}$ en comarcas de Almería, Granada, Albacete, Jaén y el Noroeste de Murcia (Caravaca de la Cruz, Moratalla, Bullas, Cehegín y Calasparra) (IGP, 2020).

La ganadería extensiva, y por ende la trashumante, genera productos de calidad y aporta beneficios ambientales. El pastoreo racional, además de permitir el aprovechamiento de recursos naturales con razas autóctonas, contribuye a la conservación de la biodiversidad (pastizales y fauna asociada) y ayuda a prevenir incendios, entre otros beneficios ecosistémicos (Garzón, 2017; MAPA, 2013; Puntas, 2019; Ramo, 2018). En nuestro entorno, como ejemplos de especies protegidas destacan la avutarda y otras aves esteparias (Comarca del Noroeste), aves necrófagas, entre las que destaca el quebrantahuesos (Sierra de Segura) o el lince ibérico (Sierra Morena). Estas dos últimas especies, en vías de extinción y protegidas merced a proyectos LIFE, tienen en el rebaño ovino un importante sustento al aprovechar sus cadáveres o depredar sobre sus corderos tras la paridera, respectivamente. A la lista de beneficios ambientales de la ganadería extensiva se asocia una serie de ventajas como la obtención de carne, leche, lana y pieles de gran calidad, rusticidad y escasos tratamientos y residuos ambientales, lo que incide sobre el medio ambiente y la calidad de los productos, sin olvidar su efecto sobre la despoblación, pues la obtención de recursos de manera sostenible de zonas despobladas contribuye a la fijación de la población al medio rural. En un artículo de opinión, Casares y Salguero (2019) reflexionan sobre las ventajas de la trashumancia y cada uno de los 17 Objetivos del Desarrollo Sostenible (ODS) de la agenda 2030, tan en boga en el debate institucional, y exponen que "la trashumancia contribuye en mayor o menor medida a todos los ODS excepto al de vida submarina (al menos de forma directa)".

A pesar de sus fortalezas, la ganadería ovina extensiva, representa a un sector en crisis por el desplome del consumo y las importaciones desde otros países. Así, el consumo de carne de cordero y cabrito en España está en serio retroceso, a pesar de los esfuerzos del sector por fomentar el consumo interno, y en 2018 (con 1,36 kg por persona y año) sólo superó al de conejo y fue casi diez veces menor que el de pollo (MAPA, 2019). Por otra parte, las enfermedades de impacto sobre la salud pública han sido un reto y un elemento limitante para el sector ovino, en especial la campaña de lucha contra la brucelosis ovina, frente a la que se han invertido cuantiosos recursos desde 1976. Sin embargo, hoy en día, nuestro nivel de saneamiento es satisfactorio, figurando Murcia como "Oficialmente Indemne de Brucelosis Ovina y Caprina" y aspirando Andalucía a finalizar el 2020 sin ningún rebaño positivo confirmado (MAPA 2020). Paradójicamente, la ganadería extensiva ovina afronta su peor crisis con un óptimo estatus sanitario y, si la tendencia no cambia, podríamos ver desaparecer el ovino extensivo de nuestro entorno en las próximas décadas. 


\section{OVINO SEGUREÑO Y TRASHUMANCIA}

La condición trashumante del ovino de la Sierra de Segura está condicionada por su marcada estacionalidad climática, cuyos pastizales pueden ser aprovechados durante el verano (agostaderos), pero deben abandonarse en otoño en busca de recursos pastables en zonas más templadas, debido a las condiciones extremas de sus inviernos, especialmente en la zona de mayor aprovechamiento herbáceo, los Campos de Hernán Perea. Este desplazamiento se realiza mayoritariamente a pie, por vías pecuarias (VV.PP.), hacia pastizales de las dehesas de Sierra Morena (invernaderos). El municipio de mayor entidad trashumante es Santiago-Pontones, donde se censaron en la última campaña de trashumancia un total de 36.493 ovinos, 1273 caprinos, 746 bovinos y 54 équidos procedentes de 68 ganaderías, que iniciaron su salida hacia las zonas de invernada entre el 10 de octubre y el 30 de diciembre de 2019 (J. Ruiz, J. comunicación personal, 20 de enero de 2020). Los trashumantes de la Sierra de Segura, junto con los de la Serranía de Cuenca y los Montes Universales (Teruel), constituyen los mayores contingentes de ganaderos que mantienen la trashumancia viva en nuestro país. Ambos núcleos de trashumantes coinciden en las zonas de invernada (mayoritariamente dehesas de Sierra Morena) y como fundamentales diferencias destaca la mayor duración del desplazamiento medio de los trashumantes aragoneses (casi un mes) frente a una semana de los trashumantes jienenses. Por otra parte, mientras que una mayoría de los trashumantes aragoneses se desplazan en camiones, el 56\% de los segureños (38) aún se desplazan a pie. Por último, mientras que los trashumantes aragoneses desarrollan la tradicional trashumancia Norte-Sur, los trashumantes andaluces de la Sierra de Segura mantienen viva una original trashumancia Este-Oeste.

El fenómeno atávico de la trashumancia, por su valor para la salvaguardia del patrimonio cultural de nuestro país, ha sido protegido en 2017 por el Ministerio de Cultura mediante el reconocimiento de "Manifestación Representativa del Patrimonio Cultural Inmaterial" (RD 385/2017), destacando que "la actividad ganadera trashumante ha aunado históricamente el aprovechamiento de los recursos naturales y el ganado, produciendo interrelaciones familiares, sociales, económicas, patrimoniales y biológicas y modelando y contribuyendo a la cohesión y vertebración del paisaje peninsular." Asimismo, en diciembre de 2019, la UNESCO incluyó a la trashumancia en la Lista Representativa del Patrimonio Cultural Inmaterial de la Humanidad y entre otros argumentos destacó que "los pastores de rebaños trashumantes poseen profundos conocimientos sobre el medio ambiente, el equilibrio ecológico y el cambio climático porque la trashumancia es uno de los métodos de crianza del ganado más sostenibles $y$ eficaces" (UNESCO, 2020).

Además, el valor cultural de la trashumancia se ve potenciado por la red de VV.PP. de nuestro país, herencia de la hegemonía de la Corona de Castilla en el comercio mundial de la lana durante siglos y cuyo patrimonio, único en el mundo, está protegido por la Ley (3/1995). Sin embargo, esta protección legal, que las considera "inembargables, imprescriptibles e inalienables" no ha evitado la alarmante destrucción de VV.PP. en nuestro país (Villavilla, 2015). Solo en la Región de Murcia existen 2.750 kilómetros lineales y 7.360 hectáreas que, además de contener otras infraestructuras como abrevaderos y descansaderos, nos conectan con el pasado histórico de los trashumantes que, desde los Montes Universales, accedían al Levante a través del Cordel de los Valencianos. Aunque la trashumancia en Murcia desapareció en la segunda mitad del s. XX, aún podemos contemplar rebaños trashumantes de ovino segureño por VV.PP. en la provincia limítrofe de Jaén. Esta imponente red de VV.PP. es un fabuloso patrimonio, de dominio público, que debemos ayudar a conservar por su valor patrimonial, económico (potencial para el turismo de naturaleza y el de- 
sarrollo rural) y ambiental (Sánchez-Sánchez, 2019; Serrano, 2017), generadora de servicios ecosistémicos (Hevia y González, 2017). Sin embargo, la red de VV.PP. tiene como función principal facilitar el tránsito ganadero, por lo que es vital garantizar su uso por los rebaños que aún practican esta ganadería sostenible y que cada vez tienen más problemas para desplazarse por la destrucción de estas infraestructuras. Aunque las condiciones del s. XXI hacen la trashumancia a pie menos penosa, especialmente gracias a la sustitución de las caballerías "hateras" por vehículos de apoyo y la telefonía móvil, sigue siendo una actividad sacrificada, lo que contribuye a la falta de relevo generacional. Por ello, y en relación con la crisis de la ganadería extensiva ovina, los trashumantes a pie son el colectivo más proclive a la desaparición, lo que a su vez desprotegerá aún más la red de VV.PP. Por ello, las iniciativas para dar a conocer la trashumancia, en los lugares donde ya no se desplazan los rebaños por las VV.PP. resultan de gran interés (Garzón, 2017). Dada la variedad de temas asociados a las VV.PP., recomendamos el monográfico "Vías pecuarias, un patrimonio único” (Del Álamo Gómez, 2017).

\section{ESTUDIANTES DE VETERINARIA Y RE- BAÑOS TRASHUMANTES EN EL SUR- ESTE}

Teniendo en cuenta el valor de la ganadería extensiva y de la trashumancia, así como el potencial del ovino segureño del Sureste, se abren oportunidades para la innovación docente que pretendemos explorar. Ya existían antecedentes desde 2012, en la Facultad de Veterinaria de Zaragoza. Así, grupos de hasta ocho alumnos, con rotación semanal, tutorizados por dos profesores, son testigos de la trashumancia de otoño con ovino de raza merina variedad de los Montes Universales, que se desplaza desde Guadalaviar (Teruel) hasta Vilches en Sierra Morena (Jaén), durante 24 días y $580 \mathrm{~km}$, en un excelente ejemplo de supervivencia de la trashumancia a larga distancia y de adaptación de una variedad autóctona (Ramo et al., 2018). Complementan esta actividad con acciones formativas como recogida de muestras para realizar trabajos de fin de grado o similares y dejando constancia de esta experiencia en el blog "Con la trashumancia" (Lacasta, 2019).

Para conocer las condiciones en las que se desarrolla la trashumancia en nuestro entorno y valorar la incorporación de nuestros estudiantes de Veterinaria, suscribimos el correspondiente convenio con ANCOS, y contactamos con trashumantes con los que compartimos los últimos dos días de "vereda" de primavera, a principios de junio de 2016. Hacemos constar que coloquialmente, en el entorno trashumante, el desplazamiento a pie de pastores y rebaños, incluidos perros o caballerías, se conoce como "hacer la vereda o ir de vereda". En noviembre de 2016 hicimos una visita, ya con estudiantes, a rebaños de bovinos de Jorge Escudero, bravos y berrendos, en trashumancia por cañadas de Albacete, y en noviembre de 2017 acompañamos durante un día al grupo de Zaragoza por la cañada Real Conquense en la provincia de Ciudad Real, ya en las proximidades de Sierra Morena. Como último paso antes de plantear la actividad de forma oficial, en junio de 2018, dos profesores y dos estudiantes voluntarios acompañamos a varios rebaños trashumantes desde Castellar hasta la Sierra de Segura. Entre las dificultades encontradas, destaca la dureza del recorrido, en especial las dos jornadas y media de montaña por la Sierra de Segura, la exposición a condiciones meteorológicas adversas, la complejidad organizativa de la infraestructura de manutención y acampada de forma autónoma y evitar ser un lastre para los trashumantes, para lo que resulta imprescindible un vehículo todoterreno. Entre las fortalezas de la actividad destacamos la buena disposición de los ganaderos a colaborar, la inmediata integración de los estudiantes en la rutina diaria, las amplias posibilidades docentes que se ofrecen, la belleza 
de los paisajes y la enriquecedora convivencia entre ganaderos, profesores y estudiantes.

Con la experiencia anterior desarrollamos, durante las veredas de otoño de 2018 y 2019 , prácticas curriculares y extracurriculares con estudiantes de veterinaria de Murcia y Cardenal Herrera (UCH-CEU) de Valencia, respectivamente. Dadas las particularidades de la activi$\mathrm{dad}$, se realizaron varias reuniones para informar a los estudiantes potencialmente interesados de las condiciones de la misma, especialmente la dureza de parte del recorrido y de la exposición a las condiciones meteorológicas, las necesidades de equipamiento, rutinas diarias y otros detalles. Además, durante el curso académico 2018/2019, los alumnos de ambas universidades conocieron la experiencia de sus compañeros en el "Debate sobre Trashumancia y Vías Pecua- rias" celebrado en la Universidad de Murcia, así como en las I Jornadas Internacionales CEU sobre trashumancia. Además, antes de realizar las prácticas, los estudiantes tuvieron conocimiento por escrito de las condiciones en las que se desarrollan (Anexo I). Durante ambos periodos se completaron 17 días de "vereda", participando cuatro profesores y dieciocho estudiantes de cinco nacionalidades (españoles, italianos, suecos, franceses y mejicanos). Durante esos periodos se obtuvieron imágenes y videos que sentaron las bases de una exposición fotográfica y un documental (Tabla 1). Con el material fotográfico, se confeccionó una exposición de 76 imágenes que se tituló "La Vereda de los Pontoneros", en alusión a la toponimia con la que denominan, en Sierra Morena, a los trashumantes de la Sierra de Segura. Además, en el entorno de un

Tabla 1. Detalles de la exposición fotográfica y el documental sobre la trashumancia en la Sierra de Segura realizados como actividades de innovación docente durante 2018 y 2019.

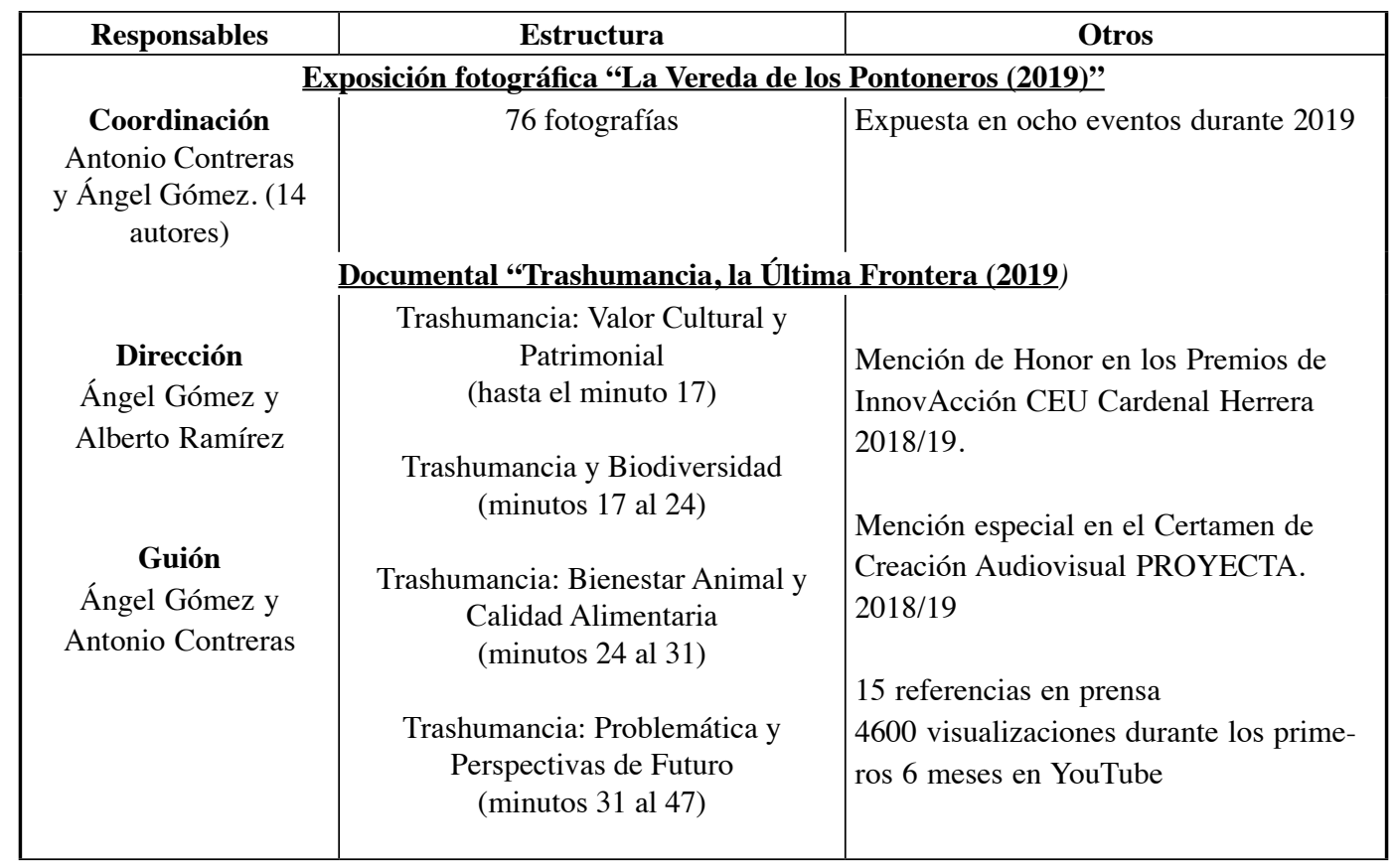

Más información: Trashumancia e Innovación Docente (2020)

https://www.um.es/web/sanidadderumiantes/practicas-con-ganaderias-trashumantes 
proyecto de innovación educativa de la Universidad de Murcia (2019-2020) con profesores de las facultades de Veterinaria, Biología y Letras, se confeccionará un catálogo de esta exposición fotográfica actualizada. Por otra parte, durante el curso 2018/2019, desarrollamos un proyecto de innovación docente (UCH-CEU) de apoyo a la trashumancia para la elaboración de cortos documentales que fueron grabados por estudiantes de comunicación audiovisual, en colaboración con alumnos de veterinaria y supervisados por profesores de ambas universidades. El documental (Trashumancia, la Última Frontera, 2019), son 47 minutos divididos en cuatro bloques.

\section{Encuadre de las prácticas con trashumantes en la programación docente de la Facultad de Veterinaria de Murcia}

Inicialmente, esta actividad se gestionó dentro de las opciones de prácticas extracurriculares del Grado de Veterinaria, coordinadas con el Centro de Orientación e Información de Empleo (COIE) y en la actualidad están encuadradas dentro del rotatorio de prácticas tuteladas (curriculares). De esta forma, la empresa externa (ANCOS) ampara la cobertura formativa permitiendo compartir la trashumancia durante una media de 7 días (variable en función de los ganaderos seleccionados) y permite la formación con sus técnicos en las diferentes instalaciones de la asociación durante la segunda semana. Los estudiantes pueden alojarse en Huéscar en la "Granja de Los Morales", de la Diputación de Granada, donde se encuentra el Centro de Sementales y se desarrolla el esquema de selección de la raza, asistir con sus técnicos al cebadero y al matadero de la IGP Cordero Segureño, así como a diferentes explotaciones del entorno de Huéscar. Con esta cobertura, los estudiantes conocerán la gestión del ovino trashumante, la mejora genética del ovino segureño, su cebo y la comercialización de sus productos.

La duración de la vereda depende del calendario de cada año y no se puede concretar de antemano con qué ganaderos se realizará, pues programan su salida en función del estado de los pastizales de las fincas de origen y destino, lo que depende fundamentalmente de las condiciones meteorológicas (lluvias en Sierra Morena y nieve en los Campos de Hernán Perea). Habitualmente, entre la tercera semana de noviembre y la primera de diciembre hay trashumantes en ruta, lo que es compatible con las fechas en las que se realiza el rotario del primer cuatrimestre. Dadas las condiciones de la trashumancia, cada grupo se compondrá de cuatro estudiantes y un profesor tutor y necesitará un vehículo todoterreno de apoyo.

La realización de actividades docentes fuera de las instalaciones universitarias obliga a considerar específicamente los riesgos asociados. En este sentido, la naturaleza de la actividad docente vinculada a los movimientos del ganado trashumante plantea distintos retos que abarcan desde los desplazamientos (en vehículo o a pie), la logística del alojamiento y manutención en condiciones adecuadas, así como los posibles riesgos biológicos derivados de la convivencia con los animales y el medio ambiente. Dada la naturaleza curricular de estas prácticas, los estudiantes se encuentran cubiertos por el seguro escolar incluido en la matrícula. No obstante, dada las particularidades de la actividad, se informa a los estudiantes de los riesgos específicos (zoonósicos y otros) tanto verbalmente como por escrito (Anexo I).

\section{Potencial docente de las prácticas con ovino segureño trashumante}

Al margen de las consideraciones sobre el potencial docente de la ganadería extensiva y la trashumancia ya indicadas, hemos detectado hasta 43 competencias que podrían desarrollarse con estas prácticas (Tabla 2). Corresponden tanto a competencias transversales de la Universidad de Murcia, como generales y específicas del Grado de Veterinaria, que se diferencian en competencias disciplinares (saber), profesio- 
Tabla 2. Competencias susceptibles de reforzar durante las dos semanas de prácticas curriculares con la Asociación Nacional de Criadores de Ovino Segureño (ANCOS) como empresa externa.

\begin{tabular}{|c|c|c|}
\hline \multicolumn{3}{|c|}{ COMPETENCIAS } \\
\hline \multicolumn{2}{|c|}{$\begin{array}{c}\text { Transversales } \\
\text { de la Universidad de Murcia } \\
\text { (CTUM) }\end{array}$} & CTUM 3, CTUM 4, CTUM 5, CTUM6 \\
\hline \multicolumn{2}{|c|}{ Generales del Título de Veterinaria (CGT) } & $\begin{array}{l}\text { CGT2, CGT3, CGT6, CGT7, CGT8, CGT10, } \\
\text { CGT11, CGT12, CGT13, }\end{array}$ \\
\hline \multirow{3}{*}{ Específicas de Veterinaria } & $\begin{array}{l}\text { Disciplinares (saber) } \\
\text { (CED) }\end{array}$ & $\begin{array}{l}\text { CED1, CED2, CED3, CED7, CED8, CED 10, } \\
\text { CED11, CED12, CED13, CED14, CED15. }\end{array}$ \\
\hline & $\begin{array}{c}\text { Profesionales } \\
\text { (saber hacer) } \\
\text { (CEP) }\end{array}$ & $\begin{array}{l}\text { CEP1, CEP2, CEP4, CEP5, CEP6, CEP8, } \\
\text { CEP9, CEP10, CEP11, CEP12, CEP16. }\end{array}$ \\
\hline & $\begin{array}{l}\text { Académicas } \\
\text { (saber ser) } \\
\text { (CEA) }\end{array}$ & $\begin{array}{l}\text { CEA1, CEA2, CEA3, CEA4, CEA5, CEA6, } \\
\text { CEA7, CEA8. }\end{array}$ \\
\hline
\end{tabular}

Más información: Descripción del Grado en Veterinaria (2020) https://www.um.es/web/veterinaria/contenido/estudios/grados/veterinaria/descripcion\#competencias

nales (saber hacer) y académicas (saber ser). La descripción detallada de las competencias a las que hacemos referencia, están disponibles online (Descripción del Grado en Veterinaria 2020). El complementar una semana de trashumancia con otra de estancia en las instalaciones de ANCOS abre el abanico de actividades a desarrollar, en el entorno específico del ganado ovino segureño. Además, el interés que profesores de otros centros de la Universidad de Murcia han mostrado en la trashumancia nos ha llevado a plantear un proyecto interdisciplinar de innovación educativa, que se desarrollará durante 2020 , para explorar otras vertientes de este potencial docente.

En relación a los contenidos de mayor interés veterinario destacan los relacionados con la producción y la sanidad animal, sin olvidar los derivados de la comercialización del cordero segureño (recental, fundamentalmente). En función de la especialización de los tutores, los estudiantes pueden ser orientados hacia una temática u otra, pero las dos semanas les permiten integrar todo el ciclo productivo del cordero en su entorno natural, en contacto con los trashumantes, y a veces también con sus familias. Toda la experiencia formativa quedará plasmada en un informe que, tras finalizar las prácticas, los estudiantes deben presentar a los tutores para su evaluación.

Como contenidos transversales se enfatizan los aspectos epidemiológicos relacionados con la transmisión de enfermedades, especialmente zoonosis. Los rebaños trashumantes están más expuestos a contagios que el resto de los rebaños extensivos. Así, al compartir con otros rebaños las VV.PP. e infraestructuras relacionadas (abrevaderos y descansaderos), en un estrecho margen temporal (otoño o primavera), se condiciona la transmisión indirecta de patógenos, por lo que la comunidad de rebaños trashumantes termina siendo una "macrounidad epidemiológica". Por otra parte, el riesgo de transmisión de patógenos entre las poblaciones de vida libre y los rebaños trashumantes es similar a la del resto de rebaños en extensivo durante el agosta- 
dero y la invernada, con la salvedad de que los trashumantes interaccionan con poblaciones de dos ecosistemas diferentes. Estas condiciones tan especiales requieren de abordajes específicos para el control de las enfermedades, por lo que el conocimiento de esta singularidad por parte de los futuros veterinarios podrá ser de utilidad para el sector. Ese conocimiento es necesario dada la dificultad de adaptación de algunas normativas a la idiosincrasia trashumante, como la necesidad de disponer de dos registros de explotaciones ganaderas (REGA), explotaciones en las que sólo pasan la mitad del año, o que durante "la vereda" no hay animales en ninguna de las dos explotaciones para las que tienen el registro, lo que supone una "anomalía administrativa". Con relación a los requisitos antes de salir de trashumancia, además de obtener la preceptiva "Guía de Origen y Sanidad Pecuaria", los ganaderos deben comunicar a las autoridades correspondientes, con diez días de antelación, la fecha y hora prevista a la que van a cruzar por lugares donde se necesita el apoyo de los agentes municipales o de la policía de tráfico (poblaciones o cruce por carreteras). Esta gestión se complica ante desplazamientos entre comunidades autónomas, con normativas diferentes, aunque esta situación no afecta a la mayor parte de trashumantes de la Sierra de Segura.

En relación con la transmisión de zoonosis, estas prácticas permiten reforzar los mecanismos de protección de los estudiantes, por lo que estos riesgos deben conocerse y se utilizan como contenidos de aprendizaje. Los protocolos de bioseguridad son más complicados de mantener en explotaciones comerciales, a diferencia de en instalaciones universitarias, y la mayoría de los casos de zoonosis descritos en estudiantes de veterinaria están asociados a actividades externas (Sánchez et al., 2017). Por ello, el estado sanitario de los ganados trashumantes y la actitud activa de los estudiantes para autoprotegerse frente a zoonosis son elementos a considerar de forma específica. Afortunadamente, la erradicación en la

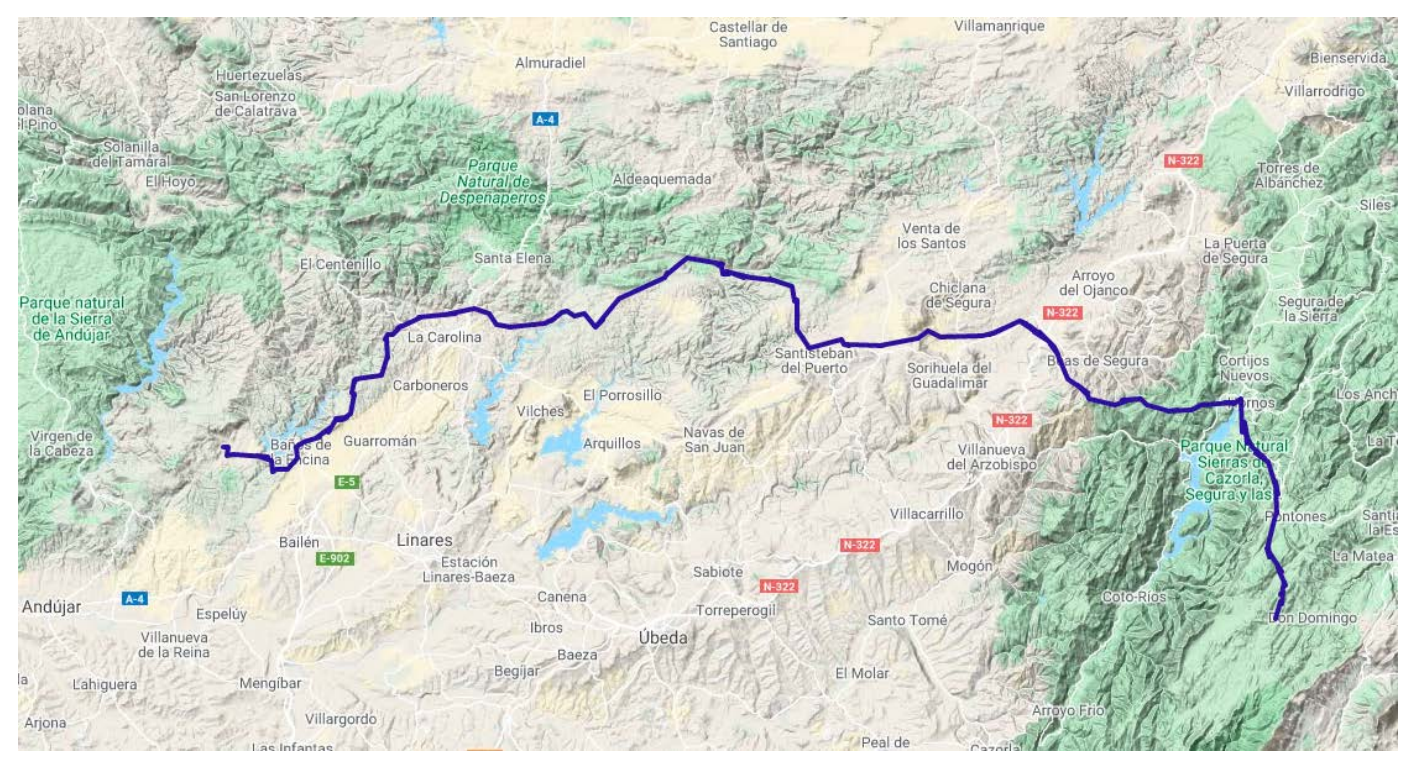

Figura 1. Una de las rutas trashumantes realizadas ( $242 \mathrm{~km}$ en 10 días), georeferenciada a través de los datos obtenidos con los collares provistos de emisores GPS (Mapa realizado a través de WTracks). 


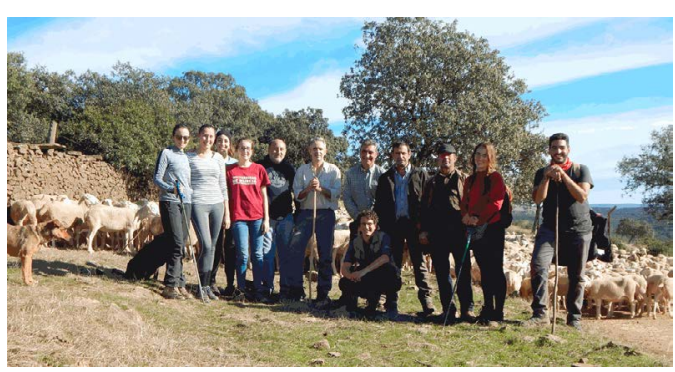

Figura 2. Algunos participantes en la trashumancia de otoño de 2018, en Sierra Morena.

práctica de la brucelosis de los pequeños rumiantes representa un salto cualitativo en la bioseguridad frente a otras épocas en la que dicha enfermedad era una de las comunicadas con mayor frecuencia en estudiantes de veterinaria (Sánchez et al., 2017). Por el contrario, la ausencia de programas nacionales de lucha frente a la fiebre $Q$ genera un escenario de incertidumbre que obliga a considerarla como uno de los principales riesgos y conocer sus determinantes, entre los que destaca la posibilidad de eliminación prolongada, tras el parto, de Coxiella burnetti, incluso a partir de hembras asintomáticas (secreciones vaginales, orina y heces). El carácter endémico de la fiebre Q en España (de declaración obligatoria en humanos desde 2015), se corresponde, en los cuatro últimos años, con la mayor notificación de casos humanos de la Unión europea (EFSA, 2019). Por ello, deben conocerse las consecuencias de esta enfermedad, especialmente en mujeres gestantes, personas inmunocomprometidas y otros grupos de riesgo (Million et al., 2015). Las infecciones transmitidas mediante picaduras de garrapatas como la borreliosis (enfermedad de Lyme), la fiebre exantemática y otras suponen riesgos que deben conocerse. Por ello, es necesario aplicar pautas de prevención de picaduras de garrapatas y controlar las normas de actuación ante una picadura: extracción adecuada de la garrapata lo antes posible, con pinzas de borde romo, des-

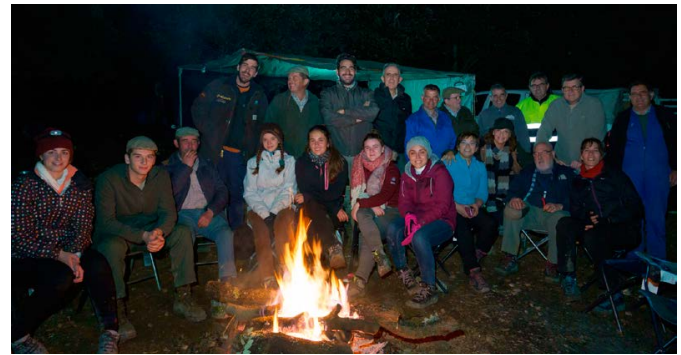

Figura 3. Algunos participantes en la trashumancia de otoño de 2019, en Sierra de Segura. Imagen cortesía de Catalina Gómez.

infección y auto-observación durante 32 días (MSCBS , 2016). En este sentido en otoño hay menor riesgo de picaduras de garrapatas que en primavera, tanto por la menor presencia de ectoparásitos en el entorno, como por una mayor protección del calzado y ropa de abrigo. Igualmente, ante la hipotética presencia $C$. burnetti en restos aerosolizados de heces, en otoño suele haber menor riesgo de transmisión aerógena pues el rebaño levanta menos polvo a su paso que al final de la primavera.

Al margen de contenidos específicamente veterinarios, destacan elementos patrimoniales relacionados con las VV.PP., sus abrevaderos y descansaderos, infraestructuras como el puente mocho (s.II) en Beas de Segura (Figura 4), monumentos naturales como las huellas de Arcosaurios en Santisteban del Puerto (icnitas de más de 230 millones de años) o diferentes manifestaciones de la arquitectura popular. Cabe destacar que parte de esta ruta coincide con el camino histórico de San Juan de la Cruz, entre Beas y Caravaca, que acaba de ser señalizado y se encuentra ampliamente documentado, tanto por Giménez (2019) como en la web (Camino de San Juan de la Cruz, 2020). Tanto las VV.PP. como la cultura trashumante y el patrimonio cultural y religioso se integran en este tramo en consonancia con el valor cultural inmaterial de la trashumancia, que justifica su declaración como "Manifestación Representativa del Patrimonio Cultural Inmaterial", tanto a nivel 


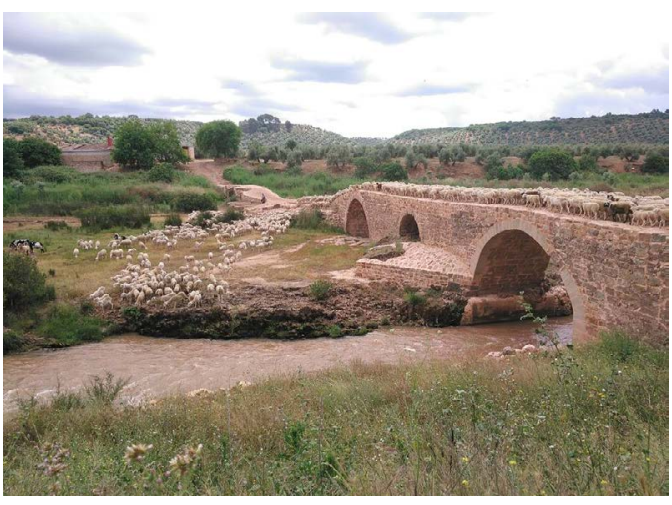

Figura 4. Las Vías Pecuarias tienen un componente cultural que transciende el puramente ganadero. Rebaño de ovino segureño trashumante cruzando por el puente mocho (s.II), en el conocido como "Camino de los Cartagineses"

nacional (Real Decreto 385/2017) como internacional (UNESCO, 2020).

Otra consideración es la contribución de estas prácticas al conocimiento y defensa de las VV.PP. El desplazamiento desde los Campos de Hernán Perea se hace por diferentes VV.PP. que confluyen en el Cordel de Hornos el Viejo, entre las que destaca el Cordel del Nacimiento del Segura, que pasa junto al emblemático nacimiento del río Segura. Entre Hornos y Beas, destaca la Vereda del Puente Mocho a Natao, y en las proximidades de Sierra Morena los trashumantes transitan por la Cañada Real Conquense, la última de las grandes rutas de la trashumancia Norte-Sur que se mantiene activa y que también utilizan los trashumantes de la Serranía de Cuenca y de los Montes Universales. Los estudiantes son testigos de las múltiples dificultades a las que se enfrentan los trashumantes cuando las VV.PP. han sido cortadas por vallados, destruidas por construcciones, carreteras o diferentes infraestructuras (aeródromo de Beas de Segura, por ejemplo), o invadidas por los olivares o el cereal, a pesar de su protección legal, e incluso a pesar de estar algunas de ellas correctamente amojonadas. Especialmente problemática es la existencia de vallas inadecuadas que invaden la vía pecuaria, responsables de lesiones o pérdida de animales, así como el desvío del agua para uso agrícola particular (abrevadero de Cañada Morales).

Participar en el acompañamiento de cientos de ovejas, manejadas por dos o tres pastores, y un par de perros careas, por VV.PP. ancestrales entre dos ecosistemas tan interesantes como la Sierra de Segura y Sierra Morena es, en sí mismo, un objetivo que justificaría esta actividad. La convivencia durante las largas jornadas (de hasta $25 \mathrm{~km}$ diarios) permite el conocimiento directo del manejo tradicional del ganado y acercarse a la cultura pastoril, sus ritos y leyendas. Este contacto humano se extiende, en ocasiones, a los habitantes de algunos pequeños pueblos o caseríos que salen a recibir a los trashumantes y a veces a ofrecerles unos dulces, un trago de mistela, o algo de fruta, o incluso a los conductores que quedan inmovilizados por el rebaño cuando una carretera ha ocupado el lugar de la vía pecuaria, y los vehículos se ven obligados a respetar la prioridad de paso del ganado. A ese respecto hemos elaborado un folleto (Figura 5) para interactuar con los conductores (Figura 6) y difundir las particularidades de la ganadería trashumante.

Al llegar al destino diario, normalmente antes de la puesta del sol, se inicia el periodo de mayor interacción entre estudiantes, profesores y ganaderos. A pesar de la fatiga por el constante caminar, se hace necesaria la cooperación para acondicionar el rebaño para la noche se hace necesaria la cooperación para acondicionar el rebaño para la noche, atender a algún animal enfermo o herido, alimentar a perros y caballerías, preparar el campamento y la cena, compartir algún aperitivo y una cerveza mientras va cayendo la tarde, y el frío comienza a hacerse protagonista. Si la lluvia no lo impide, las largas veladas otoñales, en los magníficos parajes ya descritos, generan un ambiente especial que suele prolongarse tras la cena y que permanecerá como uno de los mejores recuerdos de la "vereda". Una vez ge- 


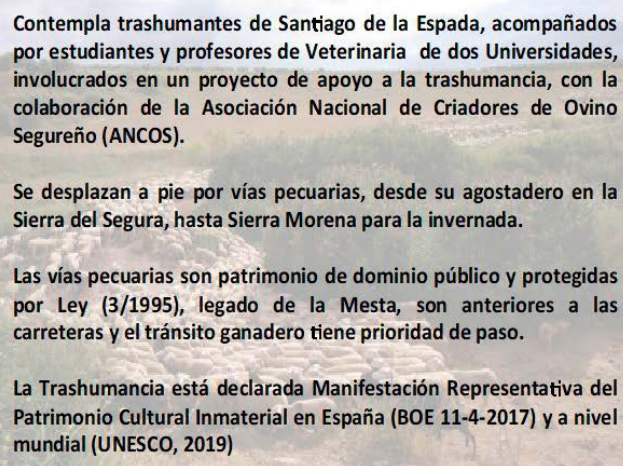
por estudiantes y profesores de Veterinaria de dos Universidades, involucrados en un proyecto de apoyo a la trashumancia, con la colaboración de la Asociación Nacional de Criadores de Ovino Segureño (ANCOS).

Se desplazan a pie por vías pecuarias, desde su agostadero en la Sierra del Segura, hasta Sierra Morena para la invernada.

Las vías pecuarias son patrimonio de dominio público y protegidas por Ley (3/1995), legado de la Mesta, son anteriores a las carreteras y el tránsito ganadero tiene prioridad de paso.

La Trashumancia está declarada Manifestación Representativa del Patrimonio Cultural Inmaterial en España (BOE 11-4-2017) y a nivel mundial (UNESCO, 2019)

No se impaciente. La vida de los trashumantes es bastante dura y gracias a ellos se mantiene una ganadería excepcional, sostenible y de alta calidad, con razas autóctonas, que contribuyen al mantenimiento de las vías pecuarias, el paisaje, la biodiversidad y la lucha contra los incendios.

Disfrute del privilegio de contemplar un sistema ganadero ancestral, que puede desaparecer en pocas décadas.

Cuando adquiera cordero o cabrito, exija productos autóctonos criados en condiciones respetuosas con el entorno.

Más información

https://www.um.es/web/sanidadderumiantes/contenido/ ganaderia-extensiva- $y$-trashumante

Figura 5. Anverso y reverso del folleto informativo distribuido a los ocupantes de los vehículos retenidos por los rebaños trashumantes.

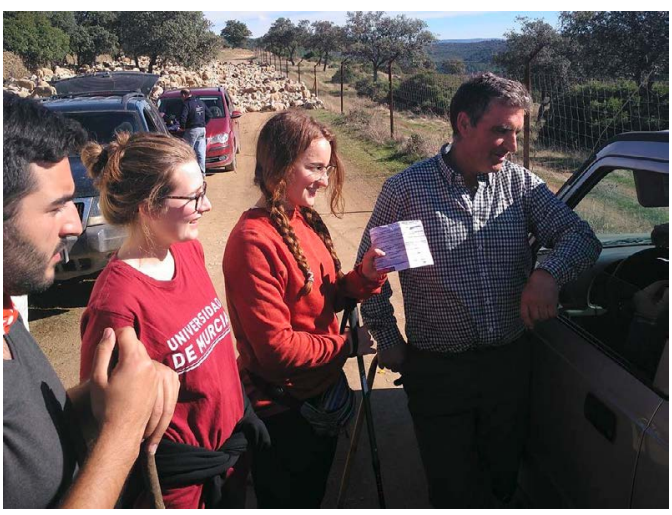

Figura 6. Interactuando con los conductores que ven interrumpido su desplazamiento en las vías pecuarias, debido a la prioridad del tránsito ganadero.

nerado este ambiente resulta de gran facilidad establecer conversaciones de interés común, y que suelen incidir en la crisis del sector ovino y los bajos precios del cordero, pero que también permiten comentar otros aspectos relacionados con enfermedades, alimentación, etc., que resultan de interés. Esta experiencia contribuye a desarrollar habilidades sociales de los futuros egresados, tan necesarias para el desempeño de la profesión.

Desde el punto de vista académico, esta actividad supone el desarrollo genuino de apren- dizaje inmersivo, de la que ya hay estudios aplicados al ámbito de la docencia en veterinaria. Así, valorando la enseñanza de la medicina de animales de abasto en granjas bovinas, Foster, Sylvester y Schoenfeld-Tacher (2018) mantienen que la experiencia inmersiva genera un conocimiento más permanente e incrementa la confianza y habilidades profesionales de los estudiantes, lo que influencia su desarrollo profesional a largo plazo.

Nuestra deuda a los trashumantes que nos facilitan estas prácticas nos obliga a corresponder en la medida de lo posible. En ese sentido, hemos valorado positivamente el efecto hacia los ganaderos de la interacción con profesores y estudiantes. El hecho de verse acompañados con personas interesadas en conocer su trabajo, ver reconocida y valorada la complicada actividad trashumante, y romper su rutina y aislamiento habitual, ha permitido generar, desde las primeras "veredas" una buena atmósfera que nos ha animado a continuar la experiencia. Las discusiones durante las veladas sobre temas profesionales (manejo reproductivo, sanitario, comercialización, etc.) han sido otro refuerzo positivo y el hecho de haber participado, además de españoles, estudiantes de otras nacionalidades han enriquecido aún más la experiencia. Por nuestra parte, 


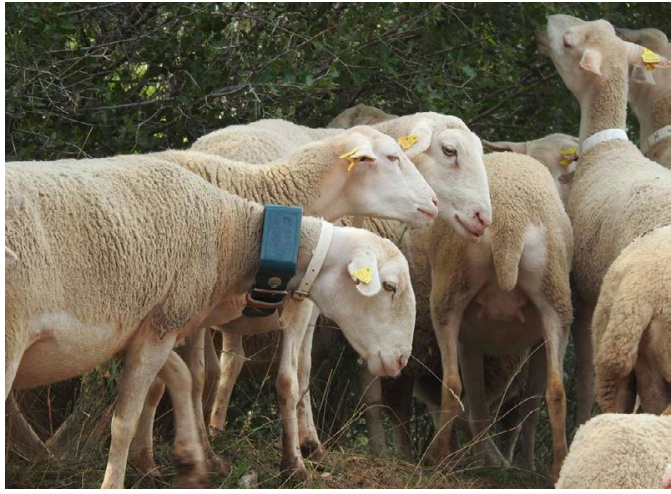

Figura 7. Oveja segureña trashumante con collar emisor de localización a través de GPS.

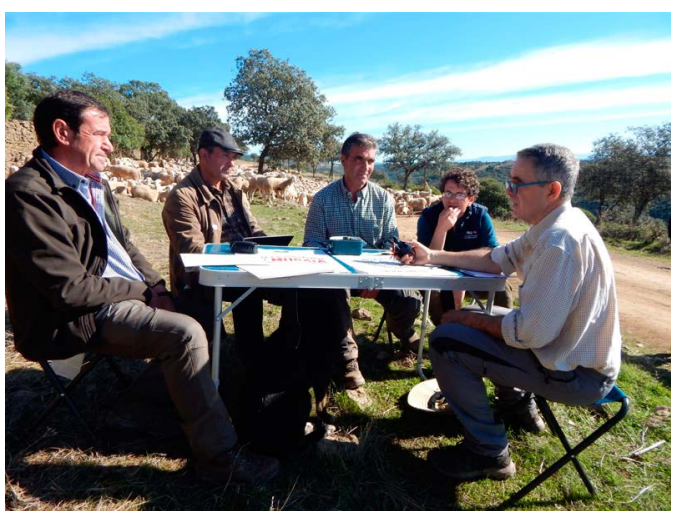

Figura 8. Formación de los trashumantes en la geolocalización de sus rebaños, a través de la recepción de datos en aplicaciones de telefonía móvil.

conociendo las particularidades del manejo de los rebaños en sus condiciones de extensividad, consideramos interesante generar conocimiento relacionado con la geolocalización de los mismos. Así, instalamos collares con emisores GPS (Figura 7) en otoño de 2018 en cinco rebaños para que los ganaderos se familiarizaran con el uso de esta tecnología (Figura 8) y, además, geolocalizar los itinerarios trashumantes (Figura 1), que podrían desvanecerse con la probable desaparición de la trashumancia a pie en un futuro. Durante la "vereda" asesoramos a los ganaderos en esta aplicación en sus teléfonos móviles y, aunque inicialmente algunos se mostraron reacios a esta tecnología, enseguida se familiarizaron con el sistema al comprobar su utilidad en la mejora de sus rutinas diarias, por lo que están contribuyendo a generalizar su uso entre otros ganaderos de Santiago-Pontones.

\section{CONCLUSIONES}

El desarrollo de prácticas curriculares con ganaderos de ovino segureño que practican la trashumancia a pie es una interesante oportunidad para la facultad de Veterinaria de Murcia, y un ejemplo de innovación docente basado en el aprendizaje inmersivo. La integración de la trashumancia con la formación ofertada por los técnicos de la Asociación Nacional de Criadores de Ovino Segureño (ANCOS), incluyendo los de la Identificación Geográfica Protegida (IGP) del Cordero Segureño, ofrece a los estudiantes un panorama integral de la mejora genética, producción, cebo y comercialización del cordero segureño. El formato de dos semanas de prácticas tuteladas, durante el primer cuatrimestre, permite la adaptación de esta actividad al calendario académico.

\section{AGRADECIMIENTOS}

A los trashumantes de la Sierra de Segura con los que hemos compartido veredas: Benito Rico, Daniel García García, Daniel García Rico, Domingo García, Félix Palomares, Jorge Escudero, Juan Trujillo, Mariano Ahibar, Nicolás López, Marisol García, y Tomás Jiménez, por permitirnos desarrollar esta experiencia, enseñarnos y brindarnos su amistad.

A José Puntas y resto del personal de ANCOS (Bernardo, Félix, Marian y Lola) por su apoyo permanente al proyecto. A Francisco Javier Ruiz Gutiérrez, veterinario de la OCA en Santiago Pontones por la actualización de los datos oficiales, así como por su permanente 
apoyo y asesoramiento. A Francisco Montoro, veterinario especializado en aves necrófagas, por su asesoramiento en el papel y conservación del quebrantahuesos en la comarca de SantiagoPontones.

A los profesores de la Universidad de Zaragoza que iniciaron las prácticas de trashumancia con estudiantes de Veterinaria, especialmente a Marian Ramo y Juan José Ramos por su constante colaboración. A Jesús Garzón y la Asociación Trashumancia y Naturaleza y a José Prats, que apoyó esta iniciativa en su inicio desde la OCA del Noroeste.

Este trabajo se ha realizado en el contexto del Proyecto de Innovación Docente "Potencial Docente de la Trashumancia, Manifestación Representativa del Patrimonio Cultural Inmaterial" (Unidad de Innovación de la Universidad de Murcia, 2019)

\section{REFERENCIAS}

BERMÚDEZ SÁNCHEZ, J. 2017: El dominio público pecuario. Ambienta, 120: 112-123.

CAMINO DE SAN JUAN DE LA CRUZ. 2020: Recuperado de https://caminodesanjuandelacruz.org/. Fecha de acceso 20 de enero de 2020.

CASARES, B., SALGUERO, C. 2019: Trashumancia y el impacto a los Objetivos de Desarrollo Sostenible. Contrainformación. es. Septiembre de 2019. Recuperado de https:/contrainformacion.es/trashumanciay-el-impacto-a-los-ods/. Fecha de acceso 20 de enero de 2020.

DEL ÁlAMO GÓMEZ, M. (Ed.). 2017: Vías pecuarias, un patrimonio único. Ambienta, 120: 1-144.

DESCRIPCIÓN DEL GRADO EN VETERINARIA. 2020. Recuperado de https://www.um.es/web/veterinaria/ contenido/estudios/grados/veterinaria/ descripcion\#competencias. Fecha de acceso 11 de enero de 2020.

EFSA(EUROPEAN FOOD SAFETY AUTHO-
RITY). 2019. SCIENTIFIC REPORT ON THE EUROPEAN UNION ONE HEALTH 2018 ZOONOSES REPORT. 2019: EFSA Journal 2019;17(12):5926, 276 pp.

FOSTER, D.M., SYLVESTER, H.J, SCHOENFELD-TACHER, R. M. 2018. Evaluation of an Immersive Farm Experience to Teach and Attract Veterinary Students to Food-Animal Medicine. Journal of Veterinary Medical Education 5(1):64-75.

GARZÓN HEYDT, J. 2017: 25 años recuperando la trashumancia en España. Ambienta, 120: $42-57$.

GIMÉNEZ MARTÍNEZ, L. 2019: Por Caminos de Trashumancia con San Juan de la Cruz. Natursport. Murcia. 99 págs.

HEVIA, V. Y GONZÁLEZ, J., 2017: El valor de las vías pecuarias como reservorios de biodiversidad y generadoras de servicios de los ecosistemas. Ambienta, 120:58-67.

HERRÁIZ SERRANO, O., 2017. Las vías pecuarias como recursos ambientales. Ambienta, 120:88-101.

IGP (INDICACIÓN GEOGRÁFICA PROTEGIDA) CORDERO SEGUREÑO. 2020: Recuperado de https://www.igpcorderosegureno. com. Fecha de acceso 20 de enero de 2020.

LACASTA D. 2019: Blog "Con la trashumancia". Recuperado de http://conlatrashumancia.blogspot.com/. Fecha de acceso 16 de enero de 2020.

LEY 3/1995, DE 23 DE MARZO, DE VÍAS PECUARIAS. 1995: BOE. 71, de 24 de marzo de 1995.

MILLION M, RAOULT D. 2015: Recent advances in the study of $\mathrm{Q}$ fever epidemiology, diagnosis and management. Journal of Infection. 2015;71:S2-S9.

MINISTERIO DE AGRICULTURA, ALIMENTACIÓN Y MEDIO AMBIENTE (MAPA). 2013: Libro Blanco de la trashumancia en España. Ministerio de Agricultura, Alimentación y Medio Ambiente (Ed.) Madrid, 217 págs.

MINISTERIO DE AGRICULTURA, PESCA Y 
ALIMENTACIÓN (MAPA), 2019. Informe del Consumo Alimentario en España 2018. 538 págs. Ministerio de Agricultura, Pesca y Alimentación (Ed.) Madrid 2019.

MINISTERIO DE AGRICULTURA, ALIMENTACIÓN Y MEDIO AMBIENTE (MAPA). 2020: Programa Nacional de Erradicación de la Brucelosis Ovina y Caprina (B. Melitensis.) V 2019-2020 ver 2020. 23 págs. Recuperado de https://www.mapa.gob.es/ es/ganaderia/temas/sanidad-animal-higieneganadera/programaboc2019-2020verfinal_ tcm30-500269.pdf.

MINISTERIO DE SANIDAD, CONSUMO Y BIENESTAR SOCIAL (MSCBS). 2016: Guía de Actuación ante Picadura de Garrapata. Recuperado de https://www.mscbs.gob.es/ profesionales/saludPublica/enfermedadesEmergentes/Crimea_Congo/docs/Guia_actuacion_picadura_garrapata_20161014.pdf. Fecha de acceso 15 de enero de 2020.

PUNTAS, J.A. 2019: Extensificación de los modelos productivos y su influencia en la sostenibilidad. Tierras (Ovino). 28: 82-84.

RAMO, M.A., , MONTEAGUDO, L.V., TEJEDOR, M..T., SIERRA, I. 2018: The ovine variety "Merino de los Montes Universales" and its good adaptation to traditional transhumant breeding system. Small Ruminant Research 166: 35-40.

REAL DECRETO 385/2017, DE 8 DE ABRIL, POR EL QUE SE DECLARA LA TRASHUMANCIA COMO MANIFESTACIÓN REPRESENTATIVA DEL PATRIMONIO CULTURAL INMATERIAL.2017: BOE. 86, de 11 de abril de 2017.

RED NACIONAL DE GRANJAS TÍPICAS (RENGRATI). 2018: Resultados del Ejercicio Económico de 2017 de Ovino de Carne. Ministerio de Agricultura, Pesca y Alimentación (Ed): 36 págs. Recuperado de https:// www.mapa.gob.es/es/ganaderia/temas/ produccion-y-mercados-ganaderos/sectoresganaderos/red-de-granjas-tipicas/ Fecha de acceso 14 de enero de 2020.
SÁNCHEZ-SÁNCHEZ, M. A (2019). Las Vías Pecuarias de la Región de Murcia. Valoración y Potencialidades como Recurso Turístico. Espacio, Tiempo y Forma (Serie VI. Geografía) 12: 265-284.

SÁNCHEZ, A., PRATS-VAN DER HAM, M., TATAY-DUALDE, J., PATERNA, A., DE LA FE, C., GÓMEZ-MARTÍN, A., CORRALES, JC., CONTRERAS, A. 2017: Zoonoses in Veterinary Students: A Systematic Review of the Literature. PLoS ONE 12(1): e0169534

TRASHUMANCIA E INNOVACIÓN DOCENTE. 2020. Recuperado de https://www. um.es/web/sanidadderumiantes/practicascon-ganaderias-trashumantes. Fecha de acceso 30 de enero de 2020.

TRASHUMANCIA, LA ÚLTIMA FRONTERA (Documental). 2019. Produce: Universidad CEU Cardenal Herrera. CEU Universities. Dirección: Ángel Gómez y Alberto Ramírez. Guion: Ángel Gómez y Antonio Contreras. 47 minutos.

UNESCO (Organización de las Naciones Unidas para la Educación, la Ciencia y la Cultura). 2020: Lista Representativa del Patrimonio Cultural Inmaterial de la Humanidad. Recuperado de https://ich.unesco.org/es/listas. Fecha de acceso 8 de enero de 2020.

VACAS FERNÁNDEZ, C. 2018: Evolución de la Oveja Segureña en Murcia durante el último medio siglo (1965-2015) y su posible futuro. Consejería de Agua, Agricultura, Ganadería y Pesca -CARM- (Ed): 196 págs. VERDU JR., LOBO JM., SÁNCHEZ-PIÑERO F., BELÉN GALLEGO B., NUMA C., LUMARET JP, CORTEZ V., ORTIZ AJ., TONELLI M., GARCÍA-TEBA JP., REY A., RODRÍGUEZ A., DURÁN J. 2018: Ivermectin residues disrupt dung beetle diversity, soil properties and ecosystem functioning: An interdisciplinary field study. Science of the Total Environment 618: 219-228

VILLAVILLA, H. 2015: 20 años de la Ley de Vías Pecuarias. El Ecologista. 84:28-31. 


\section{ANEXO I \\ Documento de compromiso para la realización de prácticas en empresa externa (AN- COS) con ganaderos trashumantes (curso 2019/20)}

$\mathrm{D}^{\mathrm{a}}$

con DNI. estudiante del Grado de Veterinaria de la Universidad de Murcia manifiesta que:

* Realizará la vereda como parte del rotatorio en empresa externa del curso..., que se desarrollará entre.... y en el que participarán como tutores los profesores...

* Ha sido informado que se utilizará un vehículo de apoyo de la U. de Murcia, conducido por los tutores y conoce las limitaciones de espacio en el mismo, por lo que se compromete a llevar un equipaje reducido.

* Ha sido informado de las particularidades de la actividad y asume los riesgos e incomodidades asociadas a la misma. Llevará ropa para lluvia (chubasquero y pantalón), calzado adaptado a las condiciones, e irá equipado para el frío, especialmente los dos primeros días (ropa térmica, gorro o braga de cuello y guantes), crema con protección solar y protector labial. Entre el material de aseo individual, se recomienda llevar una toalla de secado rápido y toallitas húmedas.

* Se compromete a cumplir con las disposiciones que se señalan para participar en la vereda y a seguir las recomendaciones de los profesores-tutores.

* Ha sido informado de la exposición a las inclemencias meteorológicas y la exigencia física de los desplazamientos a pie (unos $20 \mathrm{~km}$ diarios, aproximadamente), en ocasiones por áreas montañosas por las que el desplazamiento puede ser complicado, se requiere calzado adecuado y se recomienda bastón de senderismo o similar.

* Ha sido informado de los riesgos e incomodidades de la acampada y portará el equipo adecuado para condiciones de invierno de la Sierra de Segura.

* Conoce el comportamiento del ganado y evitará actitudes imprudentes o inadecuadas que asusten a los animales. La prioridad es su adecuado desplazamiento, así como respetar el horario y descanso de los ganaderos. Debemos estar listos en el momento en el que el rebaño se pone en marcha y no ser un lastre. No se cortará en ningún momento el rebaño y se debe caminar detrás o a los lados de este, sin interferir con las ovejas ni los perros carea. Hay que procurar aportar alguna ayuda, especialmente ante el paso por poblaciones o cruces y a la hora de organizar el campamento y la intendencia. Se establecerán turnos cada día para organizar el campamento, comprar lo necesario y preparar las comidas. 
* Mantendrá una actitud de colaboración con los pastores y resto del grupo y de respeto con los animales y el medio ambiente. Debemos ser respetuosos con el entorno. Para minimizar el uso de plásticos cada participante aportará plato, cubierto y vaso para su uso particular, y deberá aportar su propia navaja y una linterna frontal.

* Conoce los riesgos zoonósicos a los que puede exponerle la actividad y está dispuesto a mantener actitudes que minimicen el riesgo de contagio. La bioseguridad es un contenido transversal de esta actividad y se debe mantener una actitud profesional, tanto durante el contacto con los animales como al participar en la preparación de las comidas.

* Cumplirá con las actividades establecidas por los tutores y mantendrá una conducta colaborativa con el resto de los participantes.

* Llevará un diario para documentar el informe de evaluación de las prácticas.

* Tras la entrega del informe, hará una presentación a los tutores, en formato Pecha Kucka (6 minutos) sobre su actividad. Esta podrá ser seleccionada para su presentación, por parte de su autor, en la jornada académica sobre la trashumancia que se organice en la Facultad de Veterinaria.

* Compartirá con los tutores y resto de participantes de la U. de Murcia el material gráfico obtenido. Las publicaciones en redes sociales, que impliquen a otras personas del grupo, se canalizarán a través de los tutores y en todo momento se indicará que esta actividad corresponde a prácticas en empresa externa (ANCOS) de la Facultad de Veterinaria de Murcia.

* El material gráfico meritorio de ser presentado a algún concurso tendrá autoría colectiva y de ser merecedor de algún premio en metálico, éste se destinará a compensar los gastos originados por la vereda.

* Atenderá, en su caso, a citas para diferentes entrevistas con el objetivo de potenciar esta actividad en futuras ediciones.

Murcia, a ... de.... de 2019

Fdo.: 
